\title{
Hydrodynamic Process in the Langmuir-Blodgett Film Method
}

\author{
Wenjie Ji, Wenjing Zhao, Weibin Li, Yuren Wang, Jin Wang, and Ding Lan* \\ Cite This: https://dx.doi.org/10.1021/acs.langmuir.0c02975 \\ Read Online
}

ABSTRACT: Preparing organic coatings in a very controlled manner through the spreading of organic molecules on the water surface is one of the emphases for research in Langmuir-Blodgett (LB) technology. For preparing a homogeneous film and improving the quality of the film, it is our concern to have a deeper understanding of the dynamic process involved in spreading. Here, we present an overview of the hydrodynamic process under the influence of assisting the spreading solvent, which mainly focuses on the mechanical mechanisms of related phenomena. A typical spreading experiment of two-component mixed droplets on water substrate for the purpose of preparing LB films was carried out in this research. We perform the spreading of a liquid of silicone oil and oleic acid mixture on the horizontal surface of another immiscible deep water substrate, where the volatile silicone oil is the assisting spreading solvent with low viscosity. We find that it needs to exceed a certain critical value (60\% in our experiment) to achieve a uniform and centrosymmetric spreading process, which is a key factor for getting a homogeneous film. We observe that the evolution of a large droplet into liquid film and then into small droplets under the action of surface tension gradient in experiments. Gravity-viscous and surface tension-viscous dominate successively in the whole spreading process, with its spreading radius $r(t) \propto t^{1 / 4}$ and $r(t) \propto t^{3 / 4}$, respectively. However, we also obtain singular values of scaling exponents -0.033 and -0.180 , which is attributed to nonuniform distribution of the Laplace pressure caused by different curvatures near the capillary wave.

\section{INTRODUCTION}

Langmuir-Blodgett (LB) technology is considered to be an effective method for preparing thin films or monolayers. In this method, a Langmuir film at the air/water interface can be prepared using amphiphilic molecules, which is then transferred to a given substrate. The LB method can be applied to assembling the materials at the air-water interface by spreading a dispersion previously dissolved in an organic solvent. Besides, to complete a LB film, several steps including evaporation of solvent and compression of the monolayer are also required. Next, two transfer methods are the most common including Langmuir-Blodgett $(\mathrm{LB})^{1-4}$ and the Langmuir-Schaefer (LS $)^{5}$ methods corresponding to vertical and horizontal transfer to a substrate, respectively. The LB technique is widely used in the preparation of monolayer films of liquid-exfoliated graphene, ${ }^{5-8}$ monolayer of polymers, ${ }^{9,10}$ and nanomaterials, ${ }^{11-14}$ which have applications in functional coatings and thin-film electronics. Several conditions influence the quality of the monolayer in the LB process, such as $\mathrm{pH}$ values, ionic strength of the subphase, temperature, and the nature of the solvent. In addition, the influence of spreading on film formation is discussed in this article, which is usually driven by the surface tension gradient associated with a hydrodynamic phenomenon called the "Marangoni effect". ${ }^{15-17}$
Firstly, the ionic strength and $\mathrm{pH}$ of the subphase are important factors that researchers are interested in. It is electronegativity difference of ions that determines the interaction strength with the monolayer. For example, the interaction between $\mathrm{Cd}^{2+}$ and the monolayer is stronger because of the bigger electronegativity difference of $\mathrm{Cd}^{2+}$ compared with other ions with a smaller electronegativity difference. Langmuir film properties depend on the $\mathrm{pH}$ of the experimental environment. ${ }^{18-20}$ Besides, there is also an experimental study about the structure and stability of LB films which is closely related to the subphase $\mathrm{pH}$. A distorted rough surface films is prepared under the condition of subphase with low $\mathrm{pH}$, while the film has a smooth surface at higher subphase $\mathrm{pH}$. It is also found that the multilayer structure is more ordered and stable at a higher $\mathrm{pH}$ compared with a low subphase $\mathrm{pH}^{21}$

The second factor is temperature that physical adsorption depends on. Taking the simplest Langmuir adsorption model as an example, the lower the temperature, the larger the

Received: October 13, 2020

Revised: November 6, 2020 
adsorption capacity, because the Langmuir adsorption constant increases with decreasing temperature. Moreover, the structures of some solutes are temperature-dependent. For instance, the hydrophilic-hydrophobic structural transition of poly $(N$-isopropylacrylamide $)$ with increasing temperature was found by W. Saito et al. They study the difference between polyfilms spreading at the air-water interface from chloroform solution and that for water-spread polymer. The influence of temperature on layer thickness is analyzed in their experiment. $^{22}$

Thirdly, the nature of the spreading solvent is one of the most crucial factors. In terms of physical properties, there are two types of spreading solvents including "water-soluble volatile solvents" and "insoluble volatile solvents". Gericke et al. studied the influence of the spreading solvent on the properties of monolayers at the air-water interface, such as surface viscosity, the homogeneity of the monolayer, etc. ${ }^{23}$ For water-soluble volatile solvents, like ethanol, ethanol molecules would be preserved in the hydrophobic area. As a result, the properties of the spread film would be influenced by ethanol. Considering the solubility of ethanol, the mixtures (1hexadecanol, for example) containing ethanol are more likely to lose in the water subphase, but it leads to a more ordered film with a higher surface viscosity. ${ }^{23}$ For insoluble volatile solvents, such as hexane and chloroform solvent which is a less water miscible solvent, solute spread from chloroform is more likely to stay on the subphase compared with that from water. $^{23}$

In addition, using different solvents would lead to different structures of solute. Ringard-Lefebvre and Baszkin found that the difference in the orientation of the hydrophobic methyl groups in lactic acid at the air-water surface would lead to different interfacial structure at the air-water surface. ${ }^{24} \mathrm{~N}$. Vila Romeu et al. reported that the structure of PLA/GA at the air-water interface depends on the nature of solvents which leads to different structures in its bulk phase. ${ }^{25}$ The other situation is that the interaction between spreading solvent and solute in the subphase would also make effects on the properties of the spreading film. Related studies demonstrate the interaction between the solute (cyclodextrins) and the monolayer, which alters the monolayer arrangement at the air-water interface. ${ }^{26}$

These factors mentioned above directly determine the LB film quality, including subphase $\mathrm{pH}$, ionic strength, temperature, and the nature of the spreading solvent. However, we notice that these physical parameters are all related to mechanical parameters. For example, $\mathrm{pH}$ values and ionic strength would alter the viscosity of spreading solvents by the alteration of the monolayer configuration under the interaction between ions and the monolayer. Mechanical parameters such as density, viscosity, and surface tension are all temperature dependent. The nature of spreading solvents is a main factor, which closely links hydrodynamic process with the formation of the LB film. Firstly, faster spreading processes are attributed to a greater surface tension gradient of the solvents, which leads to a better distribution of the surface-active material at the water surface. Secondly, different solubility of solvent would lead to different flow patterns. For example, the spreading solvent containing ethanol would cause the downward flow and film loss into the subphase except for the Marangoni flow on the surface. ${ }^{23}$ Thirdly, considering the different viscosities of spreading solvents, a higher viscosity is usually related to a more ordered molecular arrangement. ${ }^{23}$
From the point of view of mechanics, the LB film formation process, especially the spreading process, can be considered as a hydrodynamic process. For the spreading process, several hydrodynamic phenomena would occur such as fingering instability, $^{27,28}$ Marangoni flow, viscous shear, capillary waves, $^{29}$ and even a vortex in the subphase, which would make effects on LB film formation. Therefore, the first task of this paper is to analyze the spreading process of LB film preparation from the perspective of hydrodynamics. However, in a process of fabricating the LB film, related hydrodynamics mechanisms are not well known and systematically studied. What needs to be pointed out here is how the series of hydrodynamics processes in Marangoni-driven spreading make an influence on film morphology and distribution, which is still an issue worth studying.

Based on the previous hydrodynamic studies, the spreading process is mainly related to the surface tension between phases and substrate depth. For describing the mechanisms of spreading, the definition of spreading coefficient is given $S=$ $\sigma_{\mathrm{w}}-\left(\sigma_{\mathrm{o}}+\sigma_{\mathrm{ow}}\right)\left(\sigma_{\mathrm{w}}, \sigma_{\mathrm{o}}\right.$, and $\sigma_{\mathrm{ow}}$ refer to air-water, air-oil, and oil-water interface tensions, respectively). We could get different wetting situations using this thermodynamics criterion. $S>0$ stands for a complete wetting, while $S<0$ means a partial wetting droplet. ${ }^{30,31}$ In the process of LB film preparation, two solvents are mixed as a spreading liquid. The spreading coefficient of one solvent may be less than 0 (called "liquid lens" or "partial wetting"), but the other solvent as assisting spreading agent is greater than 0 . A "partial wetting" droplet $(S<0)$ can be mixed with an assistant spreading solvent $(S>0)$. The mixture would spread on the substrate and be evenly dispersed on the liquid substrate for $S>0$, which is an important method to prepare the LB film.

The spreading driven by the "Marangoni effect" can be categorized into two cases according to the substrate depth with different approximations. ${ }^{29}$ The differences between the two cases mainly lies in the flow profile in the substrate. For spreading on thin films, a velocity gradient exists in the whole thin film with the liquid in the vicinity of the liquid-solid interface standing still. ${ }^{31}$ However, for spreading on deep films, the velocity gradient only exists in the viscous boundary layer but nearly no flow beneath it. ${ }^{29}$ Compared with the thin-layer substrate, the viscous resistance of the fluid in the deep-layer substrate is smaller, so the spreading rate of the deep-layer substrate is faster. ${ }^{32}$ Therefore, in a spreading process, the depth of substrate is a key factor which could make effects on the flow profile. For spreading on thin films, lubrication approximation is appropriate while the viscous boundary layer (Blasius boundary layer) approximation applies for spreading on deep substrate. ${ }^{29}$ A time $T=H_{0}{ }^{2} / \nu$ could be used to distinguish different approximation types in a hydrodynamics process, where $H_{0}$ and $\nu$ stand for the fluid depth and its viscosity, respectively. We can determine which type of approximation is suitable by comparing the spreading time $t$ and $T$. For $t \ll T$, i.e., we could think of it as a spreading on a deep substrate. Otherwise, we could think of it as a thin film regime. ${ }^{33}$ These two cases involve different flow profiles, so a reasonable control of the substrate thickness helps to quickly disperse the droplets to be spread. Considering that a thin substrate is not conducive to rapid spreading, we have adopted a deep substrate here to disperse droplets more quickly and evenly, thereby obtaining a high-quality LB film.

Here, our purpose is to analyze the related hydrodynamics processes in the LB film preparation and reveal its mechanical 


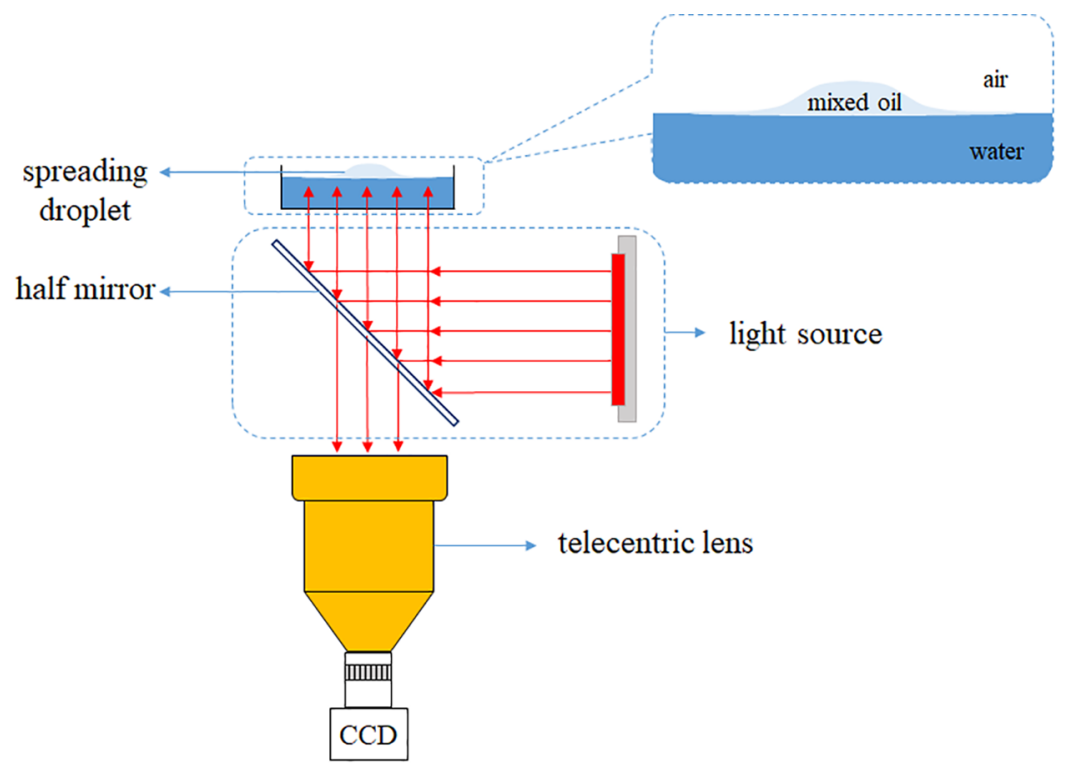

Figure 1. Experimental device for observing droplet spreading. This observation device is composed of a coaxial parallel light source, a telecentric lens, and a CCD camera. A petri dish with water is set above the observation device.
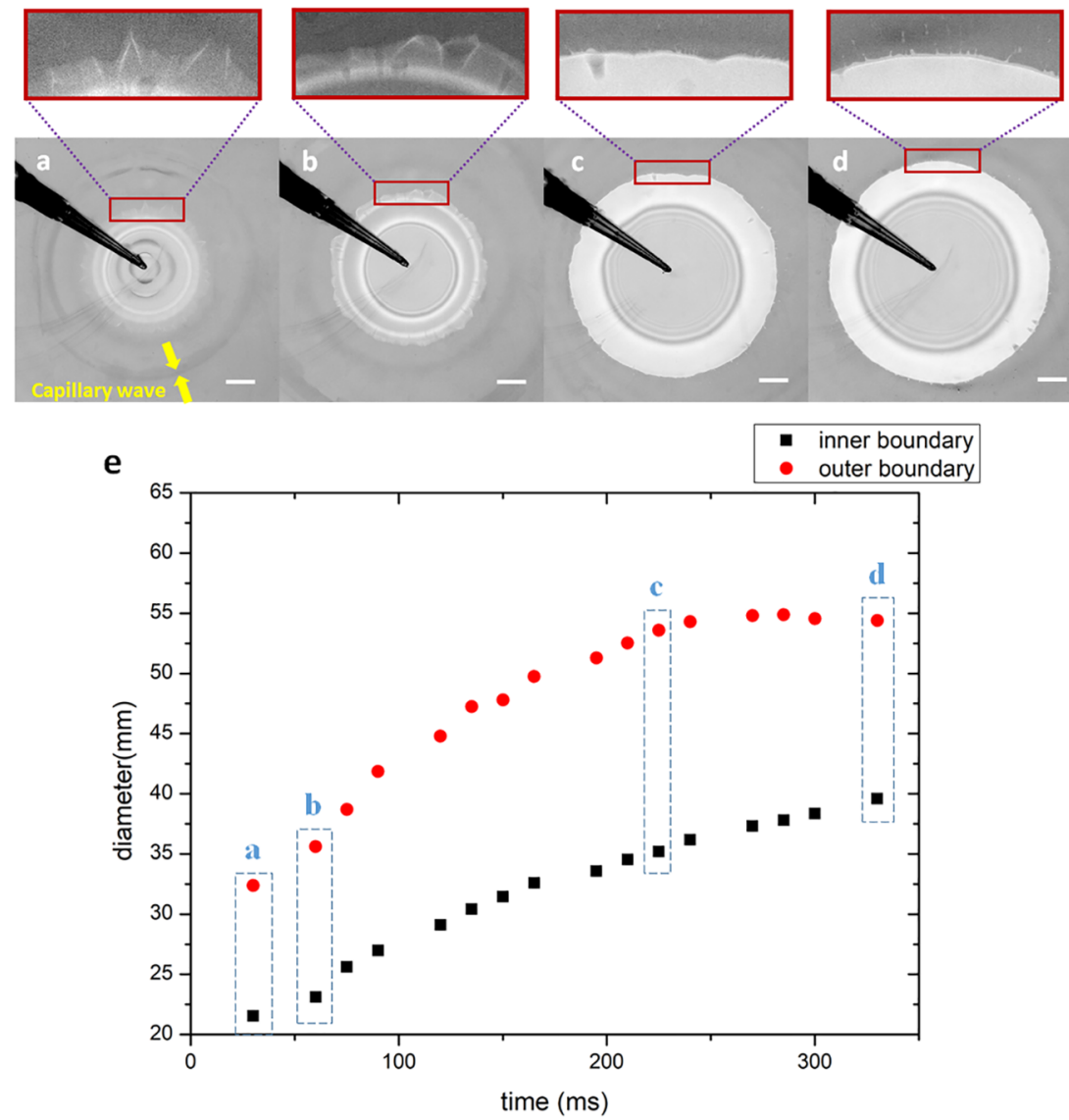

Figure 2. Successive four states (a-d) in the first spreading stage (stage 1) are shown here and outer boundary is shown in the insert. The scale bar is $10 \mathrm{~mm}$ in this picture. The positions of outer and inner boundary in the first spreading stage and the positions of a, b, c, d is marked in rectangle boxes shown in (e).

mechanism. In the hydrodynamics study, the previous experimental system is mainly built on pure liquid spreading on the other liquid substrate, which is different from the LB process where mixed liquids are usually used. Here, we introduce an assisting spreading solvent to prepare the mixed solvent droplet and perform the spreading of a liquid of silicone oil and an oleic acid mixture on the horizontal surface of another immiscible deep water substrate, and the volatile silicone oil is the assisting spreading solvent. We observed a series of phenomena including spreading, capillary wave, 


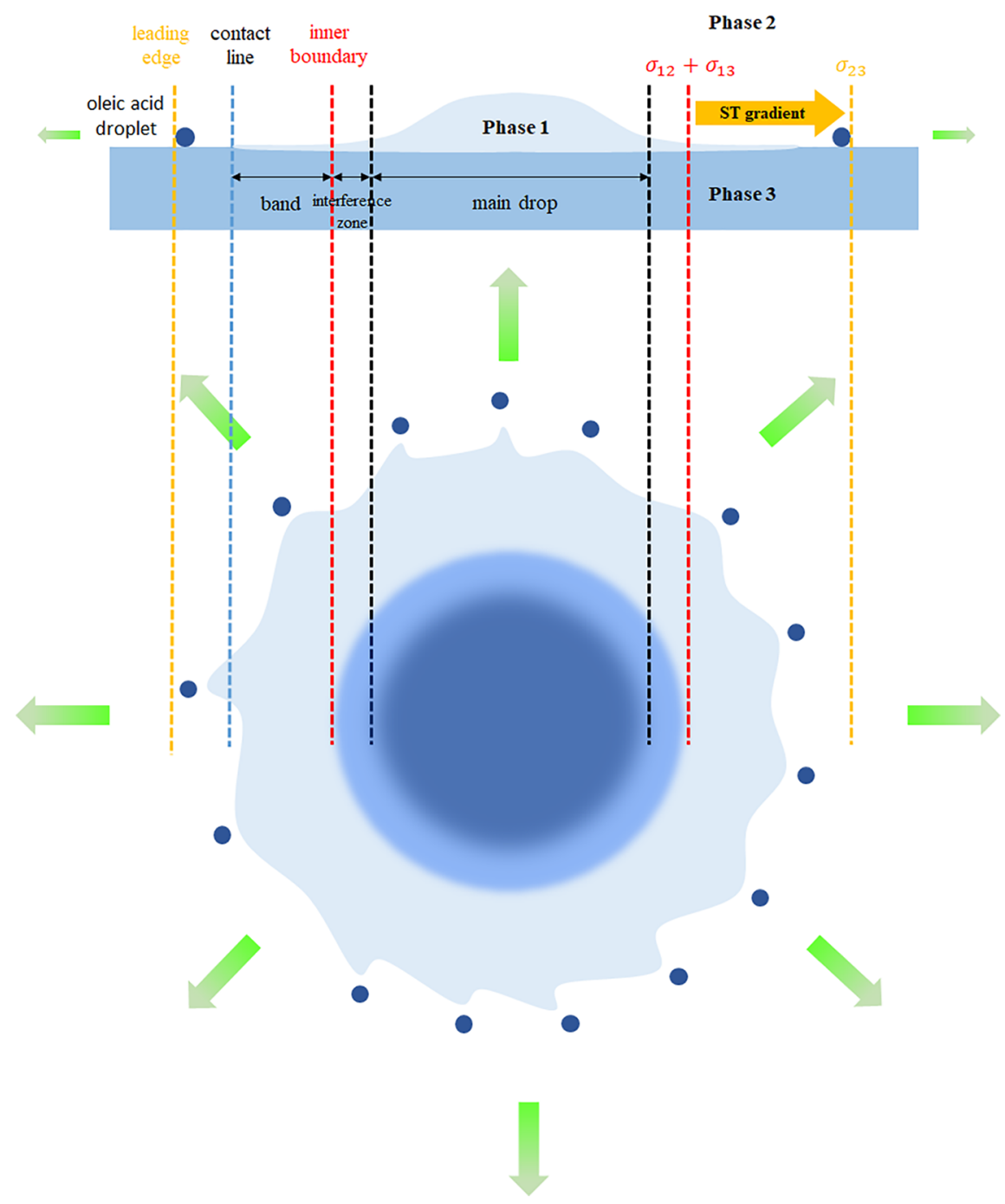

Figure 3. Schematic diagram of the drop configuration with a main drop, interference zone, band, and small emitted droplets around it. Leading edge and contact line are marked here. We show the direction and range of action of the surface tension gradient ("ST gradient") here. (Phase 1 refers to the oil phase, Phase 2 refers to the air, and Phase 3 refers to the water substrate).

instability, etc. In this paper, we give a whole scene of the hydrodynamic process in the LB method and a deep physical and mechanical mechanism.

\section{EXPERIMENTAL SECTION}

A spreading process is described by spreading coefficient $S$. As far as we know, a substance with a spreading coefficient less than 0 cannot spread on substrate. In order to spread the "partial wetting" liquid with $S<0$, we need to introduce an assisting spreading agent with $S>$ 0 . Under the effect of an assisting spreading solvent, the "partial wetting" substance can be pulled out all around along the radial direction by a surface tension gradient. After a series of hydrodynamic processes, a film composed of a "partial wetting" substance is formed. It is an important method to fabricate the LB film. However, the spreading hydrodynamics makes effects on the LB film preparation which are worthy of further experimental study.

We perform the spreading of a liquid of silicone oil and the oleic acid mixture on the horizontal surface of another immiscible deep water substrate. The volatile silicone oil is the assisting spreading solvent with low viscosity. Oleic acid (purity AR 99\%, $\rho=0.8900 \mathrm{~g} /$ $\mathrm{cm}^{3}$, surface tension $\sigma=33.8 \mathrm{mN} / \mathrm{m}$, viscosity $26 \mathrm{mP} \mathrm{s}$ at $20{ }^{\circ} \mathrm{C}$ ) was purchased from Aladdin Industrial Corporation in Shanghai China. The de-ionized water was produced by the Milli-Q Academic filtering system, Millipore Inc. Silicone oil was purchased from Shinetsu with model KF-96-0.65CS, which is highly volatile, its surface tension $\sigma=$ $15.9 \mathrm{mN} / \mathrm{m}$ at $20^{\circ} \mathrm{C}$ and an evaporation rate of $0.228 \mathrm{~g} / \mathrm{m}^{2} \mathrm{~s}$.

The oleic acid was mixed with silicone oil to obtain a twocomponent mixed liquid with different proportions. We used a pipet gun to inject the mixed drop of about $10 \mu \mathrm{L}$ softly onto the water surface with the tip as close to the surface as possible. The experimental setup is shown in Figure 1. We recorded the spreading process by the camera. In order to realize the continuous spreading process of the mixed drop, the content of the silicone oil (assisting spreading solvent) should be higher than $60 \%$. When the content of silicone oil is lower, the mixed droplet is quickly broken up into several small droplets. In this paper, we study the spreading process with a silicone oil content of $60 \%$.

\section{RESULTS AND DISCUSSION}

Marangoni-Driven Spreading Process. Marangonidriven spreading is usually realized by a surface tension gradient with a low-surface-energy substance spreading on a high-surface-energy surface. For example, surfactant solution is one of the most common low-surface-energy liquid. A surfactant molecule is amphiphilic, which is composed of a hydrophobic tail and a hydrophilic head group. After surfactant molecules adsorb onto the free surface, a monolayer forms. The surface tension depends on the distribution of surface 
concentration of surfactants. Therefore, surfactant solution will spontaneously spread until the concentration gradient is eliminated. ${ }^{34}$ The main feature of low-surface-energy substance spreading is that their surface tension is low with a spreading coefficient $S>0$, and spreading processes are driven by the surface tension gradient owing to its non-uniform distribution on the substrate surface. However, some substance would form a liquid lens on the surface of the substrate for $S<0$ but not spread. So it is a key issue of how to spread it on the surface. Here, we add an assisting spreading agent (silicone oil but not surfactant) $(S>0)$ into the substance (oleic acid) to get a mixture with $S>0$ to spread the "partial wetting" substance on the surface.

We have observed a series of phenomena in the experiment. As soon as the droplet is deposited on the surface of the water substrate, the droplet is going to form a concentric ring structure with trains of the capillary wave emerging (shown in Figure 2a). The initial state of the droplet is shown in Figure $2 \mathrm{a}$, and the structure of the droplet evolves into a double concentric ring structure, which is shown in Figure $2 b-d$. We name the two concentric rings which are distributed from the outside to the inside in Figure $2 b-d$ as "outer boundary" and "inner boundary", respectively. With the droplet spreading rapidly, the outer boundary is unstable and irregular. We observe the Plateau-Rayleigh instability of the outer boundary in the insets of Figure $2 c, d$, and small droplets were emitted outward. In similar experimental systems, the Plateau-Rayleigh instability phenomena was found before. ${ }^{38}$ Here, we observe four different forms of the outer boundary (shown in the insets of Figure 2). In Figure 2a, an unclear, jagged border appears while the droplet just touches the water surface. After $20 \mathrm{~ms}$, the boundary expands and becomes clear shown in Figure $2 \mathrm{~b}$. Until $240 \mathrm{~ms}$, new small droplets form around with the outer boundary becoming smooth in Figure $2 \mathrm{c}$,d. We recorded the position of the outer and inner boundary, and the length between the outer and inner boundary is defined as "band width" which is marked in a dotted rectangle in Figure $2 \mathrm{e}$ (points “a”, "b”, “c”, “d”). We notice that band width gradually increases from $11.5 \mathrm{~mm}$ at point "a" until $19.5 \mathrm{~mm}$ at point " $c$ " turns and it decreases from point "c" to $15.1 \mathrm{~mm}$ at point " $\mathrm{d}$ ".

According to the observation of the top view, we infer the shape of its cross section as shown in Figure 3. There is a spreading droplet which could be separated into three parts including the main drop, thickness transition region with interference fringes, and a band around the droplet (shown in Figure 3). A submicron layer or even a monolayer may exist ahead of the band according to the related reports. ${ }^{34-36}$ For the spreading at the air-liquid interface, the spreading front is often termed as a leading edge. The liquid substrate ahead of the leading edge is uncontaminated and exhibits the maximum surface tension $\sigma_{23}$. The fluid layers behind the inner boundary have a finite concentration of spreading fluid forming two new interfaces with $\sigma_{12}$ and $\sigma_{13} \cdot{ }^{29}$ By experimental observation, we give the definition of the inner boundary, which is the transition boundary from the thick bulk layer to the band, and there is no molecular interaction between the oil-water and oil-air interfaces with its net force value of $\sigma_{12}+\sigma_{13} \cdot{ }^{37} \mathrm{We}$ notice that there are interference fringes in the vicinity of the inner boundary, which shows the thickness transition from a thick layer to a thin band and is named the "interference zone", which is marked in Figure 3. It also indicates that the thickness difference of the oil film in this region is comparable to the wavelength of the light source. Ahead of the inner boundary, there is a thin band whose outer boundary is named the contact line (for convenience of description, we also call it the "outer boundary" here). It is worth noting that the outer boundary is a phase boundary of the air, oil, and water phases. However, the inner boundary is an interference fringe observed in the experiment, which means the transition of oil film thickness distribution. Considering that the instability of the oil film occurs at the contact line, there is little difference between the leading edge and the contact line in this experiment. We approximately think that the positions of the two are consistent in data analysis.

The surface tension gradient is assumed to be distributed along the band. The net force $\sigma$ of the band-covered substrate varies from a value of $\sigma_{12}+\sigma_{13}$ at the inner boundary (between the thick oil layer and the band) to $\sigma_{23}$ at the leading edge. Outside the range, the air-water surface is oil-free, which is shown in Figure 3. In Figure 2e, we notice an increase of the band width from point "a" to point " $c$ ", which is attributed to the surface tension gradient within the band. The band is stretched by the surface tension gradient from Figure 2e (point "a") to Figure 2e (point "c") with its width increasing. Next, the surface tension gradient decays significantly along with the spreading process, and the band width decreases from Figure 2e (point "c") to Figure 2e (point "d").

Scaling Relation of Spreading Diameter. Based on the analysis of evolution of spreading diameter and time, the process of spreading can be divided into five stages shown in Figure 4. We observed the four transition points in Figure 4. At

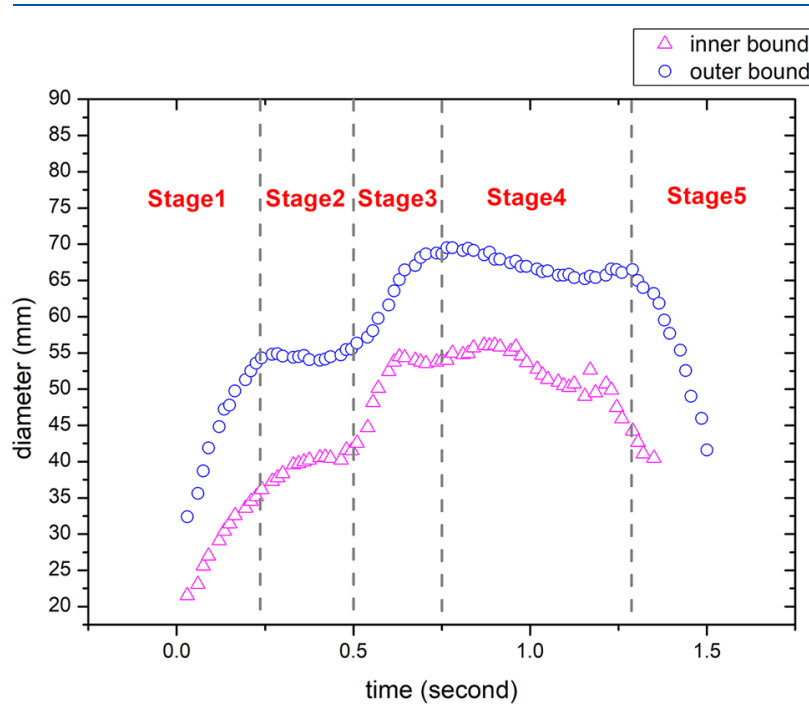

Figure 4. Five successive evolution stages after the droplet touches the surface of the substrate. The position of the inner and outer boundaries are shown here (content of silicone oil is 60\%).

transition points, the spreading speed of the diameter changes. The series of transitions are attributed to the reciprocating oscillation of the capillary wave when it is passing by. The transition point between Stage 1 and Stage 2 is attributed to the capillary wave returning back from the container wall, which compresses the contact line inward (the same reason for the transition between stages 3 and 4). The transition point between Stage 2 and Stage 3 is also caused by the capillary wave. It pushes the outer and inner boundary outward when passing by. Finally, the spreading film breaks up suddenly at the transition point between Stage4 and Stage5. 
For further analysis, we plotted the diameter of the outer boundary versus time on logarithmic scales as Figure 5, and we

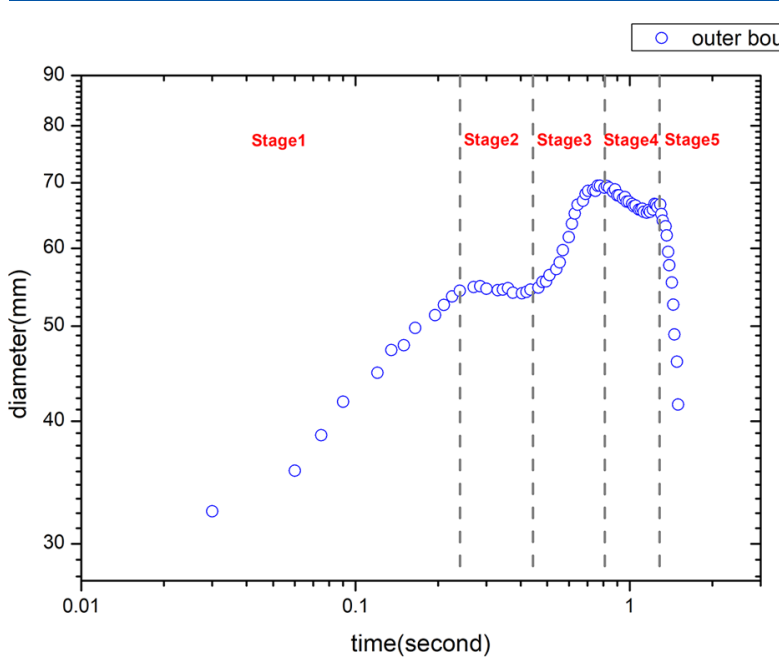

Figure 5. Scaling law of the outer boundary which is shown in double logarithmic coordinates. There are five stages in the whole spreading process. The average scaling exponents $\alpha$ for each stages according to linear fitting are $0.267,-0.033,0.751,-0.180$, and -3.05 , respectively.

observed a series of scaling laws during this spreading process. Usually, due to the interaction of various forces, physical phenomena usually occur at the same. To reveal the physical mechanism of spreading, we introduce the power law analysis. This data is fitted linearly over a finite time interval, which is convenient for us to compare it with the previous conclusion of the spreading scale. Here the diameter of the outer boundary is $D \propto t^{\alpha}$, and average exponents $\alpha$ are equal to 0.267 for Stage 1; -0.033 for Stage 2; 0.751 for Stage $3 ;-0.180$ for Stage4; and -3.05 for Stage5.

We can contrast these scaling values with existing results, where $r(t) \propto t^{3 / 4}$ (surface tension-viscous regime) and $r(t) \propto$ $t^{1 / 4}$ (gravity-viscous spreading regime) for droplet spreading on a deeply viscous substrate. The spreading is usually driven by gravity and surface tension gradients, while inertial and viscous forces are usually resistance. Here, gravity and inertial forces are volume forces that are proportional to the volume of the droplet, but surface tension and viscous force are proportional to the line length and area, respectively. At the beginning of spreading in our experiment, surface tension is negligible due to the hemispherical shape of the droplet, while inertia is also negligible for the small volume of the droplet $(10 \mu \mathrm{L})$. As a result, gravity-viscous spreading works. Then the droplet quickly spreads out into an extremely thin film, and the surface tension gradient is considered to be the main driving force and viscous as resistance with $r(t) \propto t^{3 / 4} \cdot{ }^{1-3}$

Compared with our experiment results, Stage 1 is dominated by gravity and viscous forces, but Stage 3 is the surface tensionviscous regime. Due to the capillary wave passing through, the outer boundary is pushed outward in Stage3. We notice singular values -0.033 and -0.180 for Stage 2 and Stage4, respectively, because capillary waves return from the wall and restrain the outer boundary spreading with average scaling exponents equal to -0.033 and -0.180 , respectively. In Stage5, the whole film breaks rapidly with average scaling exponents equal to -3.05 .

Capillary Wave. The capillary wave is a kind of common phenomenon in the spreading process which would influence the scale of the spreading diameter. We notice that there are two different spreading scaling laws for Stage2 and Stage4 caused by the capillary wave. In Stage2, the capillary waves are produced by the disturbance caused by the droplet touching the water surface. The capillary waves go backward from the wall of the petri dish and influence the process of spreading, which is shown in Figure 6. In the process from Figure $6 a-d$, the contact line is kept in the vicinity of the same location because of the capillary waves returning. In Stage 3 shown in Figure $6 \mathrm{e}-\mathrm{h}$, the capillary waves spread from the center of the droplet to the surroundings. When the capillary waves pass through the inner boundary and contact line, it suddenly

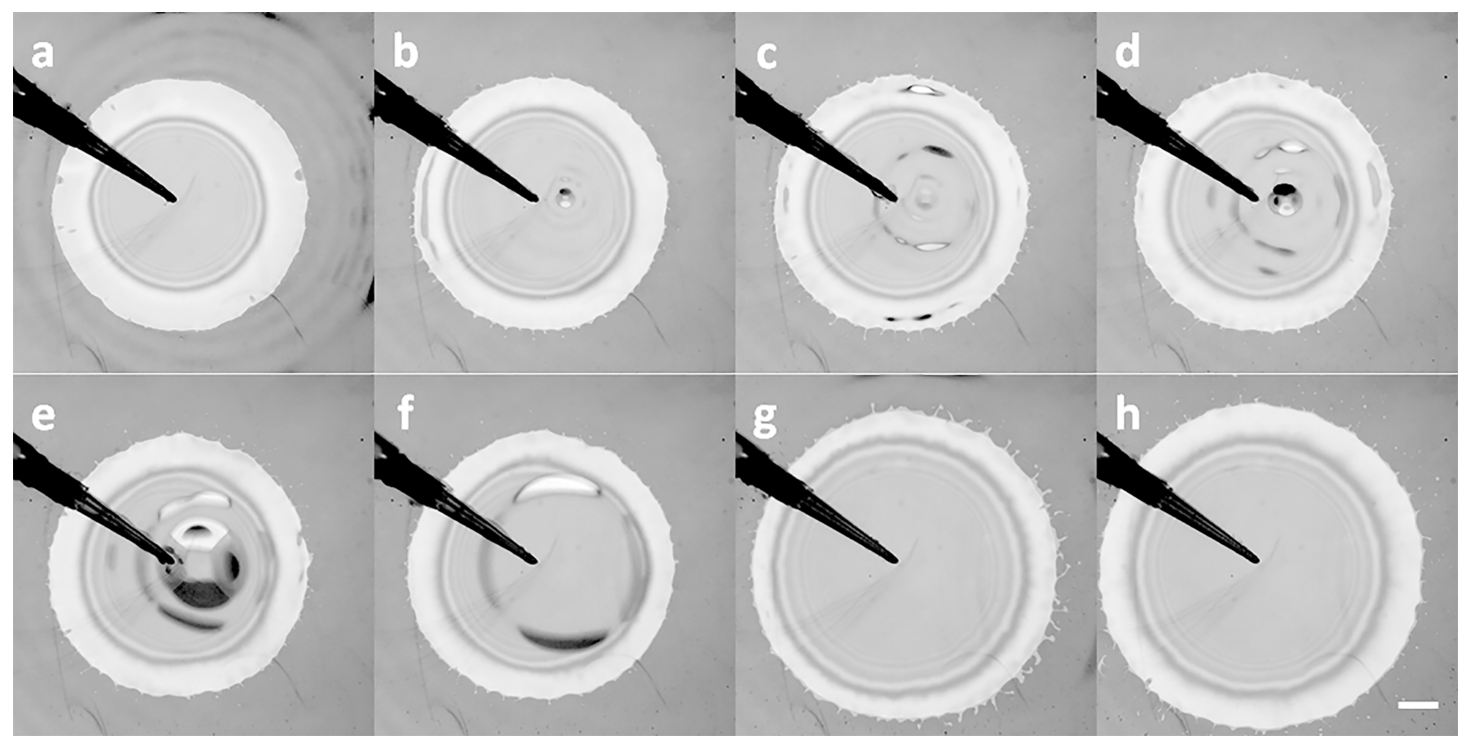

Figure 6. Process of capillary waves going through the spreading droplet. From parts a to d, the capillary waves go backward from the edge of the petri dish corresponding to Stage2. From parts e to h, the capillary waves go through the spreading droplet outward from the center corresponding to Stage3. The scale bar is $10 \mathrm{~mm}$ in this picture. 
i.
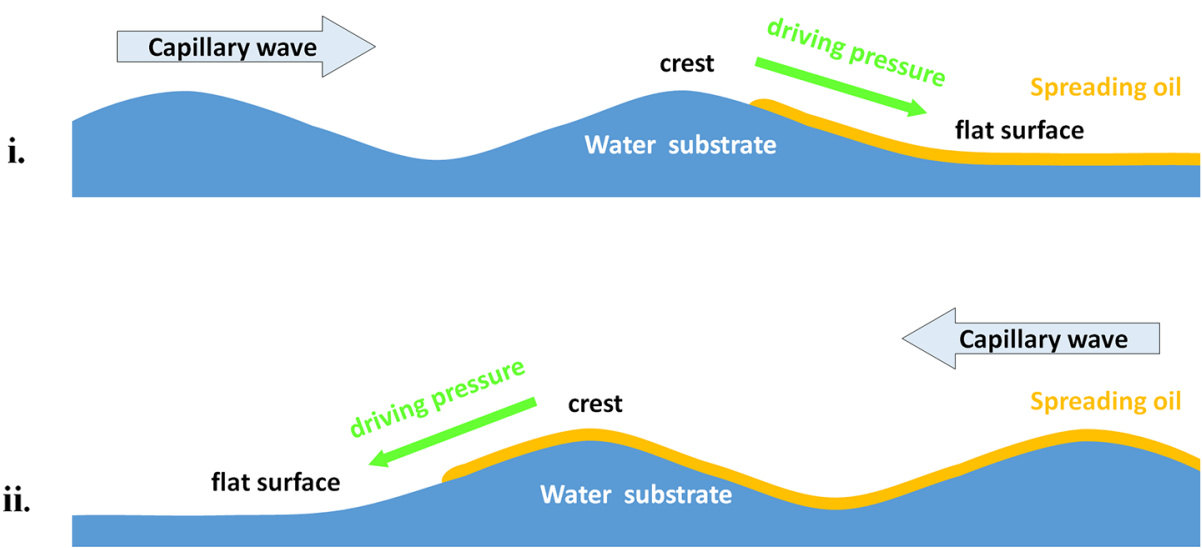

Figure 7. Distribution of Laplace pressure on the crest of the wave and on the flat surface. The directions of capillary wave propagation and direction of driving pressure are indicated here. Here, part i represents the inward direction of wave propagation, and part ii represents the outward direction of it.

expands to a large size (shown in Figure 6f,g). Similarly, when the wave returns back, the droplet retracts slightly again in Stage4. However, there is a film instability phenomenon in Stage5 shown in Figure S1 of the Supporting Information. Finally, the film breaks and disperses into several droplets.

The influence of the capillary wave on the spreading scaling laws depends on the surface curvature. The Laplace pressure is closely related to the interface curvature. According to the Young-Laplace equation, the pressure is lower under the flat surface than it is under the crest (with the curvature pointing towards the liquid substrate). ${ }^{31}$ The curvature of the crest is large, but that of the flat surface is very small (shown in Figure 7). We define the pressure difference between the crest and the flat surface as the "driving pressure". When the capillary wave passes by the contact line inward in Stage2, the driving pressure is also inward along the diameter from the crest to the flat surface (shown in Figure 7i).

Considering that the curvature of the flat surface is almost 0 , the Laplace pressure is also close to 0 . Therefore, the driving pressure between the crest and the flat surface is the same as the Laplace pressure at the crest, which could be estimated by the Young-Laplace equation $P_{c}=\frac{\sigma}{2 r} \sim 50$ pa at the crest (" $r$ " is radius of curvature on the crest in Figure 7). Similarly, when the capillary wave passes by the contact line outward in Stage3, the driving pressure is also outward along the diameter from the crest to the flat surface (shown in Figure 7ii) which pushes the contact line outward.

Here, the capillary waves are reflected inwards after reaching the periphery of the Petri dish and will cause the interface deformation when it passes by. When the capillary waves returns to the contact line, the contact line is squeezed inward under the action of driving pressure and keeps still (shown in Figure $6 \mathrm{a}-\mathrm{d}$ and Figure $7 \mathrm{i}$ ), corresponding to Stage 2 in Figure 5. The same is true of Stage4. Similarly, when the capillary wave spreads outward in Stage3, the contact line is pushed outward due to driving pressure acting on the contact line (shown in Figure $6 \mathrm{e}-\mathrm{h}$ and Figure $7 \mathrm{ii}$ ).

\section{CONCLUSION}

We have carried out a typical spreading experiment of twocomponent mixed droplets for the purpose of preparing LB films. We perform the spreading of a liquid of silicone oil and oleic acid mixture on the horizontal surface of another immiscible deep water substrate. The volatile silicone oil is the assisting spreading solvent with low viscosity. In the process of LB preparation, the concentration of assisting spreading agent is very important. It needs to exceed a certain critical value ( $60 \%$ in our experiment) to achieve a uniform spreading process, which is a key factor for getting a homogeneous film. In this article, we present an overview of the hydrodynamics process under the influence of assisting spreading solvent, which will help us to understand the process of film formation and find methods to improve its quality. At $60 \%$ concentration, we have observed the evolution of a large droplet into a liquid film and then into small droplets under the action of a surface tension gradient. The spreading is separated into five stages. The whole spreading process is dominated by gravity-viscous and surface tension-viscous, with its spreading radius $r(t) \propto t^{1 / 4}$ and $r(t) \propto t^{3 / 4}$, respectively. However, singular values of scaling exponents -0.033 and -0.180 are obtained, which is attributed to non-uniform Laplace pressure caused by curvature distribution near the capillary wave.

\section{ASSOCIATED CONTENT}

\section{(s) Supporting Information}

The Supporting Information is available free of charge at https://pubs.acs.org/doi/10.1021/acs.langmuir.0c02975.

Spreading process of the $10 \mu \mathrm{L}$ of the silicone oil-oleic acid mixed droplet (containing 60\% silicone oil), which is deposited on the water substrate (MP4)

Information on Stage5, the process of film breaking, and discussion of the effect of the depth of the substrate (PDF)

\section{AUTHOR INFORMATION}

\section{Corresponding Author}

Ding Lan - Key Laboratory of Microgravity (National Microgravity Laboratory), Institute of Mechanics, Chinese Academy of Sciences, 100190 Beijing, China; School of Engineering Sciences, University of Chinese Academy of Sciences, 100049 Beijing, China; 이이.org/0000-00019688-7731; Email: landing@imech.ac.cn

\section{Authors}

Wenjie Ji - Key Laboratory of Microgravity (National Microgravity Laboratory), Institute of Mechanics, Chinese Academy of Sciences, 100190 Beijing, China; School of 
Engineering Sciences, University of Chinese Academy of Sciences, 100049 Beijing, China; (1) orcid.org/0000-00026560-0731

Wenjing Zhao - School of Mechanical and Automotive Engineering, Qingdao University of Technology, Qingdao 266520, China

Weibin Li - Key Laboratory of Microgravity (National Microgravity Laboratory), Institute of Mechanics, Chinese Academy of Sciences, 100190 Beijing, China; School of Engineering Sciences, University of Chinese Academy of Sciences, 100049 Beijing, China; (1) orcid.org/0000-00026406-1553

Yuren Wang - Key Laboratory of Microgravity (National Microgravity Laboratory), Institute of Mechanics, Chinese Academy of Sciences, 100190 Beijing, China; School of Engineering Sciences, University of Chinese Academy of Sciences, 100049 Beijing, China

Jin Wang - School of Mechanical and Automotive Engineering, Qingdao University of Technology, Qingdao 266520, China

Complete contact information is available at: https://pubs.acs.org/10.1021/acs.langmuir.0c02975

\section{Notes}

The authors declare no competing financial interest.

\section{ACKNOWLEDGMENTS}

We thank professor Quanzi Yuan (yuanquanzi@lnm.imech.ac.cn, Institute of Mechanics, Chinese Academy of Sciences) for helpful discussions on the theory of wetting of the liquid film and reading the manuscript. We gratefully acknowledge financial support from the National Natural Science Foundation of China (Grant Nos. U1738118, 11902321, and 11472275), Strategic Priority Research Program of Chinese Academy of Sciences (Grant Nos. XDC06050200 and XDA17030X00), and Key R\&D Projects of Shandong Province (Grant No. 2019GGX102023).

\section{REFERENCES}

(1) Li, X.; Zhang, G.; Bai, X.; Sun, X.; Wang, X.; Wang, E.; Dai, H. Highly Conducting Graphene Sheets and Langmuir-Blodgett Films. Nat. Nanotechnol. 2008, 3 (9), 538-42.

(2) Kulkarni, D.D.; Choi, I.; Singamaneni, S.S.; Tsukruk, V.V. Graphene Oxide-polyelectrolyte Nanomembranes. ACS Nano 2010, 4 (8), 4667-76.

(3) Cote, L.J.; Kim, F.; Huang, J. Langmuir-Blodgett Assembly of Graphite Oxide Single Layers. J. Am. Chem. Soc. 2009, 131 (3), 10439.

(4) Szabó, T.; Hornok, V.; Schoonheydt, R. A.; Dékány, I. Hybrid Langmuir-Blodgett Monolayers of Graphite Oxide Nanosheets. Carbon 2010, 48 (5), 1676-1680.

(5) Gengler, R. Y.; Veligura, A.; Enotiadis, A.; Diamanti, E. K.; Gournis, D.; Jozsa, C.; van Wees, B. J.; Rudolf, P. Large-yield Preparation of High-electronic-quality Graphene by a LangmuirSchaefer Approach. Small 2010, 6 (1), 35-39.

(6) Fahimi, A.; Jurewicz, I.; Smith, R. J.; Sharrock, C. S.; Bradley, D. A.; Henley, S. J.; Coleman, J. N.; Dalton, A. B. Density Controlled Conductivity of Pristine Graphene Films. Carbon 2013, 64, 435-443.

(7) Kim, H.; Mattevi, C.; Kim, H. J.; Mittal, A.; Mkhoyan, K. A.; Riman, R. E.; Chhowalla, M. Optoelectronic Properties of Graphene Thin Films Deposited by a Langmuir-Blodgett Assembly. Nanoscale 2013, 5 (24), 12365-74.

(8) Matković, A.; Milošević, I.; Milićević, M.; Tomašević-Ilić, T.; Pešić, J.; Musić, M.; Spasenović, M.; Jovanović, D.; Vasić, B.; Deeks, C.; Panajotović, R.; Belić, M. R.; Gajić, R. Enhanced Sheet
Conductivity of Langmuir-Blodgett Assembled Graphene Thin Films by Chemical Doping. 2D Mater. 2016, 3 (1), 015002.

(9) Sun, F.; Castner, D. G.; Mao, G.; Wang, W.; McKeown, P.; Grainger, D. W. Spontaneous Polymer Thin Film Assembly and Organization Using Mutually Immiscible Side Chains. J. Am. Chem. Soc. 1996, 118, 1856-1866.

(10) Reitzel, N.; Greve, D. R.; Kjaer, K.; Howes, P. B.; Jayaraman, M.; Savoy, S.; McCullough, R. D.; McDevitt, J. T.; Bjørnholm, T. SelfAssembly of Conjugated Polymers at the Air/Water Interface. Structure and Properties of Langmuir and Langmuir-Blodgett Films of Amphiphilic Regioregular Polythiophenes. J. Am. Chem. Soc. 2000, $122,5788-5800$.

(11) Collier, C. P.; Saykally, R. J.; Shiang, J. J.; Henrichs, S. E.; Heath, J. R. Reversible Tuning of Silver Quantum Dot Monolayers through the Metal-insulator Transition. Science 1997, 277, 19781981.

(12) Kim, F.; Kwan, S.; Akana, J.; Yang, P. Langmuir-Blodgett Nanorod Assembly. J. Am. Chem. Soc. 2001, 123, 4360-4361.

(13) Tao, A.; Kim, F.; Hess, C.; Goldberger, J.; He, R.; Sun, Y.; Xia, Y.; Yang, P. Langmuir-Blodgett Silver Nanowire Monolayers for Molecular Sensing Using Surface-Enhanced Raman Spectroscopy. Nano Lett. 2003, 3, 1229-1233.

(14) Whang, D.; Jin, S.; Wu, Y.; Lieber, C. M. Large-Scale Hierarchical Organization of Nanowire Arrays for Integrated Nanosystems. Nano Lett. 2003, 3, 1255-1259.

(15) Fay, J. A. The Spread of Oil Slicks on a Calm Sea; Springer Science +Business Media: New York, 1969; pp 53-63.

(16) Hoult, D. P. Oil on the Sea; Springer Science +Business Media: New York, 1969.

(17) Dussaud, A. D.; Troian, S. M. Dynamics of Spontaneous Spreading with Evaporation on a Deep Fluid Layer. Phys. Fluids 1998, 10 (1), 23-38.

(18) Yazdanian, M.; Yu, H.; Zografi, G. Ionic Interactions of Fatty Acid Monolayers at the Air/Water Interface. Langmuir 1990, 6, 1093.

(19) Zasadzinski, J. A.; Viswanathan, R.; Madsen, L.; Garnaes, J.; Schwartz, D. K. Science 1994, 263, 1726.

(20) Penacorada, F.; Reiche, J.; Katholy, S.; Brehmer, L.; RodriguezMendez, M. L. Monolayers and Multilayers of Uranyl Arachidate. 1. Study of the Interaction of Dissolved Uranyl Ions with Arachidic Acid Langmuir Monolayers. Langmuir 1995, 11, 4025-4030.

(21) Penacorada, F.; Reiche, J.; Dietel, R.; Zetzsche, T.; Stiller, B.; Knobloch, H.; Brehmer, L. Monolayers and Multilayers of Uranyl Arachidate 2. Influence of the Subphase $\mathrm{pH}$ on the Structure and Stability of Langmuir-Blodgett Films. Langmuir 1996, 12, 1351-1356. (22) Saito, W.; Kawaguchi, M.; Kato, T.; Imae, T. Spreading Solvent and Temperature Effects on Interfacial Properties of Poly $(\mathrm{N}$ isopropylacrylamide) Films at the Air-Water Interface. Langmuir 1996, 12, 5947-5950.

(23) Gericke, A.; Simon-Kutscher, J.; Huehnerfuss, H. Influence of the Spreading Solvent on the Properties of Monolayers at the Air/ Water Interface. Langmuir 1993, 9, 2119-2127.

(24) Ringard-Lefebvre, C.; Baszkin, A. Behavior of Poly(D,L-lactic acid) Monolayers at the Air-Water Interface. Effect of Spreading Solvents. Langmuir 1994, 10, 2376.

(25) Vila Romeu, N.; Minones, J.; Iribarnegaray, E.; Conde, O.; Casas, M. Influence of the Solvent on the Spreading of Poly[(D,Llactic acid)-co-(glycolic acid)] Monolayers. Colloid Polym. Sci. 1997, 275, 580-586.

(26) Vila-Romeu, N.; Nieto-Suárez, M.; Broniatowski, M. The Interaction of Cyclodextrins with Phosphatidylethanolamine Langmuir Monolayers: Influence of the Spreading Solvent and Subphase Conditions. Thin Solid Films 2008, 516, 8852-8859.

(27) Saffman, P. G.; Taylor, G. I. The Penetration of a Fluid into a Porous Medium or Hele-Shaw Cell Containing a More Viscous Liquid. Proc. R. Soc. London A 1958, 245, 312-329.

(28) Bischofberger, I.; Ramachandran, R.; Nagel, S.R. Fingering versus Stability in the Limit of Zero Interfacial Tension. Nat. Commun. 2014, 5, 5265. 
(29) Berg, S. Marangoni-driven Spreading along Liquid-liquid Interfaces. Phys. Fluids 2009, 21, No. 032105.

(30) Harkins, W. D.; Feldman, A. Films. The Spreading of Liquids and the Spreading Coefficient. J. Am. Chem. Soc. 1922, 44, 26652685.

(31) Gennes, P.-G. d.; Brochard-Wyart, F.; Quere, D. Capillarity and Wetting Phenomena, 1st ed.; Springer-Verlag: New York, 2004.

(32) Borgas, M. S.; Grotberg, J.B. Monolayer Flow on a Thin Film. J. Fluid Mech. 1988, 193, 151-170.

(33) Jensen, O. E. The Spreading of Insoluble Surfactant at the Free Surface of a Deep Fluid Layer. J. Fluid Mech. 1995, 293, 349-378.

(34) DiPietro, N.D.; Huh, C.; Cox, R.G. The Hydrodynamics of the Spreading of One Liquid on the Surface of Another. J. Fluid Mech. 1978, 84, 529-549.

(35) Zisman, W. A. The Spreading of Oils on Water Part I. Ionized Molecules Having Only One Polar Group. J. Chem. Phys. 1941, 9, 534.

(36) Mercer, E.H. Spreading of Paraffin Oils Containing Fatty Acids on Aqueous Solutions of Calcium Ions. Proc. Phys. Soc. 1939, 51, $561-565$.

(37) Foda, M.; Cox, R.G. The Spreading of Thin Liquid Films on a Water-air Interface. J. Fluid Mech. 1980, 101 (1), 33-51.

(38) Wodlei, F.; Sebilleau, J.; Magnaudet, J.; Pimienta, V. Marangoni-driven flower-like patterning of an evaporating drop spreading on a liquid substrate. Nat. Commun. 2018, 9, 820. 